\section{MRS-India emphasizes advanced materials for energy applications}

www.mrsi.org.in

T he Materials Research Society of India (MRS-I) held its 24th Annual General Meeting on February 11-13, 2013 at the Indira Gandhi Centre for Atomic Research (IGCAR), Kalpakkam, Tamilnadu State. With a conference theme of "Advanced Materials for Energy Applications," the meeting witnessed talks from distinguished scientists as well as energetic interaction among the attendees.

The highlight of the inaugural session was an inspiring address by MRS-I founder C.N.R. Rao of the Jawaharlal Nehru Centre for Advanced Scientific Research, Bangalore, who outlined the distinctive position that materials research holds in interdisciplinary collaboration to attain sustainable development for humankind. MRS-I President G. Sundararajan of the International Advanced Research Centre, Hyderabad, stressed the need for materials innovations in diverse fields including energy storage and nuclear energy to solve the burgeoning global energy problem.

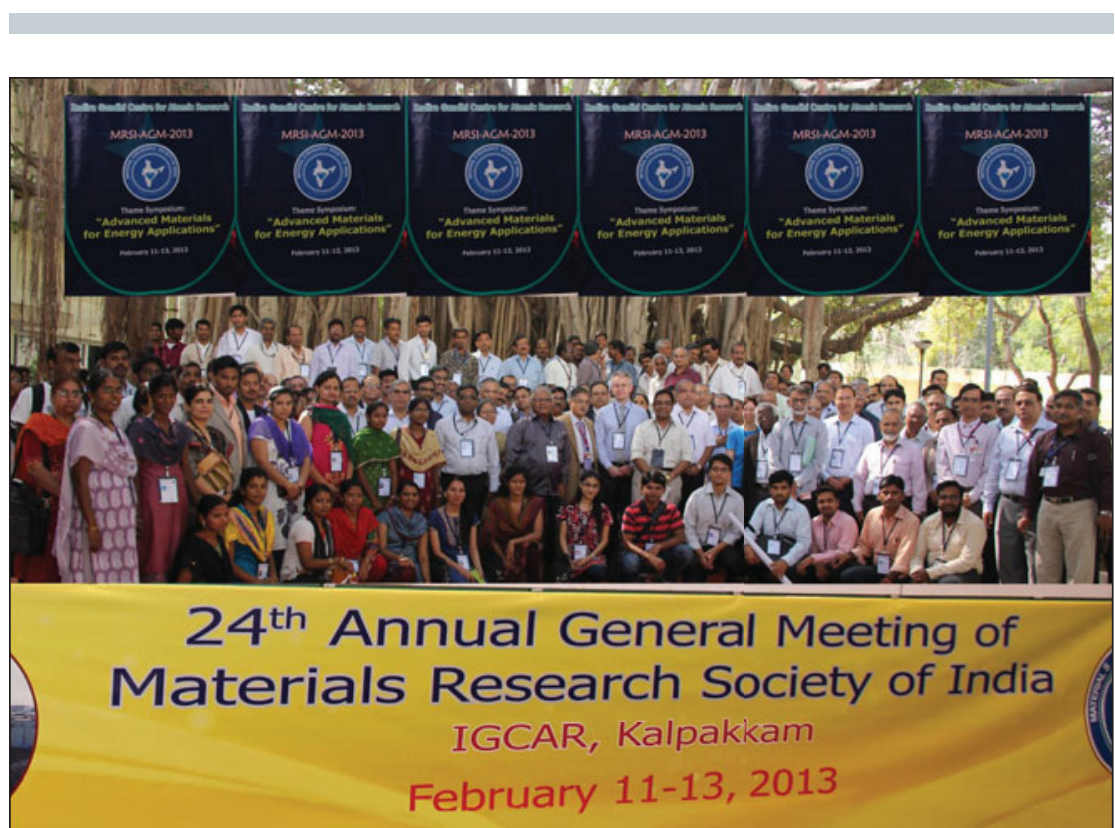
ample, M.K. Sanyal of the Saha Institute of Nuclear Physics (SINP), Kolkata, described the development of thirdgeneration synchrotron facilities enabled with high-energy photon beams and the opportunities these facilities offer for detailed probing of buried interfaces. With SINP as the nodal institute from India, Sanyal has been responsible for developing the Indian Beamline at the Photon Factory Synchroton, Japan, for Indian researchers to conduct cuttingedge research. Sanyal described success with silicon-germanium multilayers at this facility. Materials researchers in India will soon be equipped with indigenously developed advanced synchrotron generation technology with the Indus-2 project, currently under construction near Indore, Madhya Pradesh, where Sanyal is developing a surface scattering beamline.
Commenting on the upcoming commissioning of India's first indigenously designed 500 MWe Prototype Fast Breeder Reactor (PFBR) at IGCAR, nuclear scientist Srikumar Banerjee, India's former Secretary of the Department of Atomic Energy, reiterated the importance of fundamental materials research in nuclear science and engineering to achieve a transition to a closed-fuel cycle for

long-term supply of fissile materials and for developing pressure tubes with high-creep-withstanding capabilities. Though liquid sodium technology has been available for decades, the mastering of aspects of sodium cooled fast reactors by Indian engineering facilities through rigorous experimentation resulting in this important milestone was strongly praised by Banerjee.

The first day of the Meeting was also devoted to presentations by award recipients. Scalability for applications is an important metric in determining the feasibility of energy technologies such as solar cells. Organic photovoltaics (PVs), with their ability to be directly printed, are attractive from a manufacturing point of view, as discussed by Richard Friend of the University of Cambridge, United Kingdom. Friend, in his International Materials Science and Technology Award lecture, presented some of his group's achievements with $\pi$-conjugated organic polymers and heterojunctions. He also described the role of singlet electronhole excitations (excitons) for enhancing efficiencies of organic light-emitting diodes and PVs.

Suresh Das of the National Institute for Interdisciplinary Science and Technology, Thiruvananthapuram, in his MRS-I Distinguished Lectureship Award lecture, spoke on the development of functional materials based on conjugated polymeric molecules.

News coverage of this Meeting was funded, in part, by the Center for Study of Science, Technology and Policy (CSTEP), Bangalore; MRS-India; and MRS-I-Kalpakkam Chapter.

Bishal Madhab Mazumdar

Photo courtesy of IGCAR on behalf of MRS-India, Kalpakkam Chapter. 